\title{
Linear Code Implies Public-Key Traitor Tracing
}

\author{
Kaoru Kurosawa ${ }^{1}$ and Takuya Yoshida ${ }^{2}$ \\ 1 Department of Computer and Information Sciences, \\ Ibaraki University, Japan \\ kurosawa@cis.ibaraki.ac.jp \\ 2 Department of Communications and Integrated Systems, \\ Tokyo Institute of Technology, Japan \\ takuya@crypt.ss.titech.ac.jp
}

\begin{abstract}
In this paper, we first show that three public-key $(k, n)$ traceability schemes can be derived from an $[n, u, d]$-linear code $\mathcal{C}$ such that $d \geq 2 k+1$. The previous schemes are obtained as special cases. This observation gives a more freedom and a new insight to this field. For example, we show that Boneh-Franklin scheme is equivalent to a slight modification of the corrected Kurosawa-Desmedt scheme. This means that $\mathrm{BF}$ scheme is redundant or overdesigned because the modified KD scheme is much simpler. It is also shown that the corrected KD scheme is the best among them.
\end{abstract}

\section{Introduction}

In such applications as pay TV, CD-ROM distribution and online databases, data should only be available to authorized users. To prevent unauthorized users from accessing data, the data supplier will encrypt data and provide only the authorized users with personal keys to decrypt it. However, some authorized users (traitors) may create a pirate decoder.

A $(k, n)$-traceability scheme is a scheme in which at least one traitor is detected from a confiscated pirate decoder if there are at most $k$ traitors among $n$ authorized users. Chor, Fiat and Naor [4 introduced the first $(k, n)$-traceability scheme. Their scheme is, however, non-constructive. Stinson and Wei showed some explicit constructions by using combinatorial designs [10. In the above two schemes, a private-key encryption scheme is used to encrypt a session key.

On the other hand, the first public-key $(k, n)$-traceability scheme was shown by Kurosawa and Desmedt [6, Sec.5]. That is, anyone can broadcast encrypted data to authorized users. Although Shamir's $(k+1, n)$-threshold secret sharing scheme was used in their original scheme, we should use Shamir's $(2 k-1, n)$ threshold secret sharing scheme to avoid a linear attack given by [10. We call such a corrected scheme the corrected KD scheme.

After that, Boneh and Franklin presented another public-key $(k, n)$-traceability scheme [2]. Only the above two schemes are known as public-key $(k, n)$-traceability schemes currently.

In this paper, we first show that three public-key $(k, n)$-traceability schemes can be derived from an $[n, u, d]$-linear code $\mathcal{C}$ such that $d \geq 2 k+1$. We call 
them linear coded KD scheme (LC-KD scheme), linear coded BF scheme (LCBF scheme) and linear coded KD' scheme (LC-KD' scheme), respectively. The previous schemes are obtained as special cases. This observation gives a more freedom and a new insight to the study of this field.

For example, we show that Boneh-Franklin scheme (BF scheme) is equivalent to a slight modification of the corrected KD scheme. (We call it modified KD scheme. It will be given in Sec 5.3 . ) This means that BF scheme is redundant or overdesigned because modified KD scheme is much simpler. Indeed, BF scheme must use a public code matrix $\Gamma$ and $2 k$ additional secret random numbers $\beta_{1}, \cdots, \beta_{2 k}$ which modified KD scheme does not require. More generally, we prove the equivalence between LC-BF scheme and LC-KD' scheme.

We also show that LC-KD scheme is better than LC-KD' scheme from a view point of key generation. This implies that the corrected KD scheme is better than modified KD scheme from a view point of key generation. Since modified KD scheme is better than BF scheme as shown above, we see that the corrected KD scheme is the best among them.

We finally prove the secrecy and the black box traceability of LC-KD scheme under the decision Deffie-Hellman assumption. Those of LC-KD' scheme and LC-BF scheme are proved similarly. The tracing algorithm of BF scheme for any pirate decoder is obtained as a special case. (It is not written clearly in the original paper [2]. It is not written at all in their latest version [3].)

\begin{tabular}{|c|c|}
\hline Generalized Scheme & Original Scheme \\
\hline LC-KD scheme & corrected KD scheme \\
LC-KD' scheme & modified KD scheme \\
LC-BF scheme & BF scheme \\
\hline
\end{tabular}

\section{Preliminaries}

\subsection{Notation}

An $[n, u, d]$-linear code is a linear code of length $n$, dimension $u$ and the minimum Hamming distance $d$.

Let $q>n$ be a prime. Let $G_{q}$ be a group of prime order $q$. Let $g \in G_{q}$ be a generator of $G_{q}$. For example, $G_{q}$ is a subgroup of $Z_{p}^{*}$ of order $q$, where $q \mid p-1$. Alternatively, we can use an elliptic curve over a finite field.

- denotes the inner product of two vectors over $G F(q)$.

\subsection{DDH Assumption}

The decision Diffie-Hellman assumption (DDH) assumption says that no polynomial statistical test can distinguish with non negligible advantage between the two distributions $\mathbf{D}=\left(g, g^{r}, y, y^{r}\right)$ and $\mathbf{R}=\left(g, g^{r}, y, v\right)$, where $g, y, v$ are chosen at random from $G_{q}$ and $r$ is chosen at random in $Z_{q}$. 


\subsection{Model of Traitor Tracing}

In the model of traceability schemes, there are a data supplier $T$, a set of $n$ authorized users and a pirate user. Some authorized users are malicious and they are called traitors. The traitors create a pirate key $e_{p}$. The pirate key is used in a pirate decoder.

Suppose that there are at most $k$ traitors. Then a $(k, n)$-traceability scheme is a scheme such that at least one traitor is detected from a confiscated pirate decoder. A $(k, n)$-traceability scheme has four components.

Key Generation: The key generation algorithm $\mathcal{K}$ is a probabilistic polynomial time algorithm that outputs $\left(e_{T}, e_{1}, \cdots, e_{n}\right)$ on input $1^{l}$, where $l$ is the security parameter. $e_{T}$ is the broadcast encryption key of the data supplier $T$ and $e_{i}$ is the personal decryption key of authorized user $i$.

$T$ runs $\mathcal{K}$ and sends $e_{i}$ to authorized user $i$ secretly.

Encryption: The encryption algorithm $\mathcal{E}$ is a probabilistic polynomial time algorithm that takes an encryption key $e_{T}$ and a session key $s$ to return a header $h$; we write

$$
h \stackrel{R}{\leftarrow} e_{T}(s) .
$$

The data $m$ is encrypted by using a secure symmetric encryption function $E$ with the session key $s$ as $E_{s}(m)$. Finally, $T$ broadcasts $\left(h, E_{s}(m)\right)$.

Decryption: Then decryption algorithm $\mathcal{D}$ is a deterministic algorithm that takes the personal decryption key $e_{i}$ and a header $h$ to return the session key $s$; we write

$$
s \leftarrow e_{i}(h) .
$$

Each authorized user $i$ can recover $s$ from $h$ by using his personal key $e_{i}$ and then decrypt $E_{s}(m)$ to obtain the data $m$.

Tracing: $T$ can detect at least one traitor from a pirate key $e_{p}$ by using a tracing algorithm. We have black box traceability if the pirate decoder can only be used as an oracle. That is, the tracing algorithm cannot examine the pirate key $e_{p}$. For black box tracing, we shall assume that the pirate decoder is resettable to its initial state, as in [5].

In what follows, a session key $s$ is chosen from $G_{q}$.

\section{Previous Public-Key $(k, n)$ Traceability Schemes}

\subsection{Corrected Kurosawa-Desmedt Scheme}

Key Generation: The data supplier $T$ chooses a uniformly random polynomial $f(x)=a_{0}+a_{1} x+\cdots+a_{2 k-1} x^{2 k-1}$ over $G F(q)$. Then $T$ gives to each authorized user $i$ the personal decryption key $e_{i}=f(i)$, where $i=1,2, \ldots, n$. He next publishes $g$ and $y_{0}=g^{a_{0}}, y_{1}=g^{a_{1}}, \ldots, y_{2 k-1}=g^{a_{2 k-1}}$ as the public key. 
Encryption: For a session key $s \in G_{q}, T$ computes a header as $h=\left(g^{r}, s y_{0}^{r}, y_{1}^{r}, \ldots, y_{2 k-1}^{r}\right)$, where $r$ is a random number. $T$ broadcasts $h$.

Decryption: Each user $i$ computes $s$ from $h$ as follows by using $f(i)$.

$$
s=U /\left(g^{r}\right)^{f(i)}, \text { where } U=s y_{0}^{r} \prod_{j=1}^{2 k-1}\left(y_{j}^{r}\right)^{i^{j}} .
$$

\subsection{Boneh-Franklin Scheme}

BF scheme makes use of a public code matrix $\Gamma$ defined as follows. Consider the following $(n-2 k) \times n$ matrix $G$ :

$$
G=\left(\begin{array}{ccccc}
1 & 1 & 1 & \cdots & 1 \\
1 & 2 & 3 & \cdots & n \\
1^{2} & 2^{2} & 3^{2} & \cdots & n^{2} \\
\vdots & \vdots & \vdots & \ddots & \vdots \\
1^{n-2 k-1} & 2^{n-2 k-1} & 3^{n-2 k-1} & \cdots & n^{n-2 k-1}
\end{array}\right) \quad(\bmod q)
$$

Let $w_{1}, \ldots, w_{2 k}$ be a basis of the linear space of vectors satisfying

$$
G \mathbf{x}=0 \bmod q .
$$

Viewing these $2 k$ vectors as the columns of a matrix, we obtain an $n \times 2 k$ matrix $\Gamma$ :

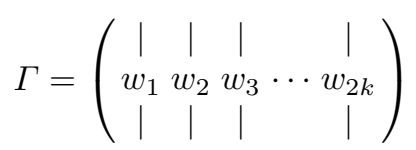

Define the code as the set of rows of the matrix $\Gamma$. Hence, it consists of $n$ codewords each of length $2 k$.

Key Generation: For $i=1, \ldots, 2 k$, the data supplier chooses a random $a_{i} \in Z_{q}$ and compute $y_{i}=g^{a_{i}}$. Then $T$ computes $z=\prod_{i=1}^{2 k} y_{i}^{\beta_{i}}$ for random $\beta_{1}, \ldots, \beta_{2 k} \in$ $Z_{q}$ and publishes $z, y_{1}, \ldots, y_{2 k}$ as the public key. The personal decryption key of user $i$ is computed as

$$
\theta_{i}=\left(\sum_{j=1}^{2 k} a_{j} \beta_{j}\right) /\left(\sum_{j=1}^{2 k} a_{j} \gamma_{j}\right) \quad(\bmod q),
$$

where $\gamma^{(i)}=\left(\gamma_{1}, \ldots, \gamma_{2 k}\right) \in \Gamma$ is the $i^{\prime}$ th codeword of $\Gamma$.

Encryption: For a session key $s \in G_{q}, T$ computes a header as $h=\left(s z^{r}, y_{1}^{r}, \ldots, y_{2 k}^{r}\right)$, where $r$ is a random number. $T$ broadcasts $h$.

Decryption: Each user $i$ computes $s$ from $h$ as follows by using $\theta_{i}$.

$$
s=s z^{r} / U^{\theta_{i}}, \text { where } U=\prod_{j=1}^{2 k}\left(y_{j}^{r}\right)^{\gamma_{j}} .
$$


Remark 3.1. In the key generation, $a_{1}, \cdots, a_{2 k}$ must be chosen so that $\sum_{j=1}^{2 k} a_{j} \gamma_{j} \neq 0 \quad(\bmod q)$ for $i=1, \cdots, n$. This was overlooked in [2].

\section{Linear Code Implies Public-Key Traitor Tracing}

This section shows that if there exists an $[n, u, d]$-linear code $\mathcal{C}$ such that $d \geq$ $2 k+1$, then three public-key $(k, n)$-traceability schemes are derived. We call them linear coded KD scheme (LC-KD scheme), linear coded BF scheme (LCBF scheme) and linear coded KD' scheme (LC-KD' scheme), respectively. The corrected KD scheme and the original BF scheme are obtained as special cases.

Let $H$ be a parity check matrix of an $[n, u, d]$-linear code over $G F(q)$ such that $d \geq 2 k+1$. Any $2 k$ columns of $H$ are linearly independent because $d \geq 2 k+1$. This property plays a central role in the proof of traceability of our schemes.

We assume that $H$ is publicly known. Note that $H$ is an $(n-u) \times n$ matrix over $G F(q)$. Let the $i$ th column of $H$ be $\mathbf{b}_{i}=\left(b_{1, i}, b_{2, i}, \cdots, b_{n-u, i}\right)^{T}$.

\subsection{LC-KD Scheme}

Assume that the first row of $H$ is $(1, \cdots, 1)$.

Key Generation: The data supplier $T$ chooses $\left(a_{1}, \cdots, a_{n-u}\right)$ uniformly at random Let $\left(e_{1}, \cdots, e_{n}\right)=\left(a_{1}, \cdots, a_{n-u}\right) H$. $T$ gives $e_{i}$ to authorized user $i$ as the personal decryption key for $i=1,2, \ldots, n$. He next publishes $y_{1}=g^{a_{1}}, y_{2}=$ $g^{a_{2}}, \ldots, y_{n-u}=g^{a_{n-u}}$ as the public key.

Encryption: For a session key $s \in G_{q}, T$ computes a header as $h=\left(g^{r}, s y_{1}^{r}, y_{2}^{r}, \ldots, y_{n-u}^{r}\right)$, where $r$ is a random number. $T$ broadcasts $h$.

Decryption: Each user $i$ computes $s$ from $h$ as follows by using $e_{i}$.

$$
s=U /\left(g^{r}\right)^{e_{i}}, \text { where } U=s y_{1}^{r} \prod_{j=2}^{n-u}\left(y_{j}^{r}\right)^{i^{j}} .
$$

The tracing algorithm will be given in Sec:7

\section{$4.2 \quad$ LC-BF Scheme}

Key Generation: The data supplier $T$ chooses $\left(a_{1}, \cdots, a_{n-u}\right)$ uniformly at random in such a way that $\left(a_{1}, \cdots, a_{n-u}\right) \cdot \mathbf{b}_{i} \neq 0$ for $i=1, \cdots, n$. The personal decryption key of user $i$ is computed as

$$
\theta_{i}=\left(a_{1}, \ldots, a_{n-u}\right) \cdot\left(\beta_{1}, \ldots, \beta_{n-u}\right) /\left(a_{1}, \ldots, a_{n-u}\right) \cdot \mathbf{b}_{i} .
$$

Next let $y_{i}=g^{a_{i}}$. Then $T$ computes $z=\prod_{i=1}^{n-u} y_{i}^{\beta_{i}}$ for random $\beta_{1}, \ldots, \beta_{n-u} \in Z_{q}$ and publishes $z, y_{1}, \ldots, y_{n-u}$ as the public key. 
Encryption: For a session key $s \in G_{q}, T$ computes a header as $h=\left(s z^{r}, y_{1}^{r}, \ldots, y_{n-u}^{r}\right)$, where $r$ is a random number. $T$ broadcasts $h$.

Decryption: Each user $i$ computes $s$ from $h$ as follows by using $\theta_{i}$.

$$
s=s z^{r} / U^{\theta_{i}}, \text { where } U=\prod_{j=1}^{n-u}\left(y_{j}^{r}\right)^{b_{j, i}} .
$$

\subsection{LC-KD' Scheme}

This is a slight modification of LC-KD scheme.

Key Generation: The data supplier $T$ chooses $\left(a_{1}, \cdots, a_{n-u}\right)$ uniformly at random in such a way that $\left(a_{1}, \cdots, a_{n-u}\right) \cdot \mathbf{b}_{i} \neq 0$ for $i=1, \cdots, n$. Let $\left(e_{1}, \cdots, e_{n}\right)=$ $\left(a_{1}, \cdots, a_{n-u}\right) H$. (Note that $e_{i} \neq 0$ for $i=1, \cdots, n$.) $T$ gives $e_{i}$ to authorized user $i$ as the personal decryption key for $i=1,2, \ldots, n$. He next publishes $y_{1}=g^{a_{1}}, y_{2}=g^{a_{2}}, \ldots, y_{n-u}=g^{a_{n-u}}$ as the public key.

Encryption: For a session key $s \in G_{q}, T$ computes a header as $h=\left(s g^{r}, y_{1}^{r}, y_{2}^{r}, \ldots, y_{n-u}^{r}\right)$, where $r$ is a random number. $T$ broadcasts $h$.

Decryption: Each user $i$ computes $s$ from $h$ as follows.

$$
s=s g^{r} / U^{1 / e_{i}}, \text { where } U=\prod_{j=1}^{n-u}\left(y_{j}^{r}\right)^{b_{j, i}} .
$$

Remark 4.1. In $h, s$ is multiplied to $g^{r}$ in LC-KD' scheme while it is multiplied to $y_{1}^{r}$ in LC-KD scheme.

\section{Relationship with the Original Schemes}

\subsection{Corrected KD Scheme}

Let $\mathcal{C}$ be an $[n, n-2 k, d]$-Reed Solomon code over $G F(q)$, where $d=2 k+1$. Then it is clear that the corrected KD scheme is obtained from LC-KD scheme as a special case.

\subsection{BF Scheme}

In BF scheme, note that $G$ (shown in Sec 3.2) is a generator matrix of an $[n, n-$ $2 k, d]$ Reed-Solomon code over $G F(q)$. Further we see that $G \cdot \Gamma=\mathcal{O}$ from eq.(1). Hence $\Gamma^{T}$ is a parity check matrix of the Reed-Solomon code $\mathcal{C}$. This implies that the original BF scheme is obtained from LC-BF scheme as a special case. 


\subsection{Modified KD Scheme}

In LC-KD' scheme, let $\mathcal{C}$ be an $[n, n-2 k, d]$-Reed Solomon code over $G F(q)$, where $d=2 k+1$. Then the following scheme is obtained. We call it modified KD scheme because it is a slight modification of the corrected KD scheme.

Key Generation: The data supplier $T$ chooses a uniformly random polynomial $f(x)=a_{0}+a_{1} x+\cdots+a_{2 k-1} x^{2 k-1}$ over $G F(q)$ such that $f(i) \neq 0$ for $i=1, \cdots, n$. Then $T$ gives $f(i)$ to authorized user $i$ as the personal decryption key for $i=$ $1,2, \ldots, n$. He next publishes $y_{0}=g^{a_{0}}, y_{1}=g^{a_{1}}, \ldots, y_{2 k-1}=g^{a_{2 k-1}}$.

Encryption: For a session key $s \in G_{q}, T$ computes a header as $h=\left(s g^{r}, y_{0}^{r}, y_{1}^{r}, \ldots, y_{2 k-1}^{r}\right)$, where $r$ is a random number. $T$ broadcasts $h$.

Decryption: Each user $i$ computes $s$ from $h$ as follows by using $f(i)$.

$$
s=s g^{r} / U^{1 / f(i)}, \text { where } U=\prod_{j=0}^{2 k-1}\left(y_{j}^{r}\right)^{i^{j}} .
$$

Remark 5.1. In $h, s$ is multiplied to $g^{r}$ in modified KD scheme while it is multiplied to $y_{1}^{r}$ in the corrected KD scheme.

\section{Equivalence}

\subsection{LC-BF Scheme = LC-KD' Scheme}

LC-BF scheme is more complicated than LC-KD' scheme because it uses secret random numbers $\beta_{1}, \cdots, \beta_{n-u}$ which LC-KD' scheme does not use. Nevertheless, we show that they are equivalent. This means that LC-BF scheme is redundant or overdesigned.

Public-key equivalence: In the key generation of LC-BF scheme, let

$$
c=\sum_{i=1}^{n-u} a_{i} \beta_{i}
$$

For any fixed $\left(a_{1}, \cdots, a_{n-u}\right)$, it is easy to see that $\operatorname{Pr}(c \neq 0)=1-(1 / q)$. Therefore, we assume that $c \neq 0$ in what follows.

The public key of LC-BF scheme is $p k=\left(z, y_{1}, \ldots, y_{n-u}\right)$. First since $q$ is a prime and $z \in G_{q}, z$ is a generator of $G_{q}$. Next note that

$$
z=\prod_{i=1}^{n-u} y_{i}^{\beta_{i}}=\prod_{i=1}^{n-u} g^{a_{i} \beta_{i}}=z^{c} .
$$

Let $a_{i}^{\prime}=a_{i} / c$. Then we have

$$
y_{i}=g^{a_{i}}=z^{a_{i} / c}=z^{a_{i}^{\prime}} .
$$


Now it is clear that $\left(a_{1}, \cdots, a_{n-u}\right) \cdot \mathbf{b}_{i} \neq 0$ if and only if $\left(a_{1}^{\prime}, \cdots, a_{n-u}^{\prime}\right) \cdot \mathbf{b}_{i} \neq 0$, where $i=1, \cdots, n$. Therefore, the public key $p k$ of LC-BF scheme is equivalent to that of LC-KD' scheme.

\section{Header equivalence: Clear.}

Decryption equivalence: In LC-BF scheme, from eq.(3), we obtain that

$$
1 / \theta_{i}=\left(a_{1}, \ldots, a_{n-u}\right) \cdot \mathbf{b}_{i} / c=\left(a_{1}^{\prime}, \cdots, a_{n-u}^{\prime}\right) \mathbf{b}_{i} .
$$

On the other hand, in LC-KD' scheme,

$$
e_{i}=\left(a_{1}, \ldots, a_{n-u}\right) \cdot \mathbf{b}_{i} .
$$

Therefore, $1 / \theta_{i}$ of LC-BF scheme is equivalent to $e_{i}$ of LC-KD' scheme.

Secrecy equivalence: The same public key and the same header are used in both schemes. Therefore, the secrecy of LC-BF scheme against outside enemies is equivalent to that of LC-KD' scheme.

Traceability equivalence: Suppose that there exists a pirate decoder $M_{0}$ for LC-BF scheme which is not (black box) traceable. Then we show that there exists a pirate decoder $M_{1}$ for LC-KD' scheme which is not (black box) traceable. Let $k$ traitors be $i_{1}, \cdots, i_{k}$ in both schemes.

Consider LC-KD' scheme in which a public key is $p k=\left(g, y_{1}, \cdots, y_{n-u}\right)$ and the private key of user $i$ is $e_{i}$. From the above equivalence, the same $p k$ is used and the private key of user $i$ is $\theta_{i}=1 / e_{i}$ in LC-BF scheme.

From our assumption, there exists an algorithm $B$ which creates an untraceable pirate decoder $M_{0}$ from $p k$ and $\theta_{i_{1}}, \cdots, \theta_{i_{k}}$ for LC-BF scheme.

Now in LC-KD' scheme, our traitors first create $M_{0}$ by running $B$ on input $p k$ and $1 / e_{i_{1}}, \cdots, 1 / e_{i_{k}}$. They then use $M_{0}$ as their pirate decoder $M_{1}$.

Finally it is easy to show that if there is a tracing algorithm which detects some traitor from $M_{1}$, then $M_{0}$ is also traceable. This contradicts our assumption. Hence, $M_{1}$ is not traceable.

The converse part is proved similarly.

Now we have proved the following theorem.

Theorem 6.1. $L C$-BF scheme is equivalent to $L C-K D$ ' scheme.

\section{2 $\quad$ BF Scheme $=$ Modified KD Scheme}

From Theorem 6.1 we have the following equivalence.

Corollary 6.1. BF scheme is equivalent to modified KD scheme.

However, BF scheme is more complicated than the modified KD scheme because it must use a public code matrix $\Gamma$ and $2 k$ additional secret random numbers $\beta_{1}, \cdots, \beta_{2 k}$. This means that $\mathrm{BF}$ scheme is redundant or overdesigned. 


\subsection{Comparison}

We compare three schemes, LC-KD scheme, LC-BF scheme and LC-KD' scheme. We have seen that LC-BF scheme is equivalent to LC-KD' scheme, and hence redundant.

Now in LC-KD' scheme, $a_{1}, \cdots, a_{n-u}$ must be chosen in such a way that $e_{i} \neq 0$ for $i=1, \cdots, n$, which LC-KD scheme does not require. This check is very inefficient if $n$ is large. Therefore. LC-KD scheme is better than LC-KD' scheme from a view point of key generation.

Similarly, the corrected KD scheme is better than modified KD scheme from a view point of key generation. Further, modified KD scheme is better than $\mathrm{BF}$ scheme as shown in Sec6.2 As a conclusion, we see that the corrected KD scheme is the best among them.

\section{$7 \quad$ Secrecy and Traceability}

In this section, we prove the secrecy and the traceability of LC-KD scheme, LC-KD' scheme and LC-BF scheme.

Note that any $2 k$ columns of $H$ are linearly independent because $d \geq 2 k+1$.

\subsection{Secrecy of LC-KD Scheme}

Theorem 7.1. LC-KD scheme is indistinguishably secure against chosen plaintext attack under the DDH assumption.

Proof. Similarly to the proof of [6, Theorem 14], we can show that the secrecy of LC-KD scheme is reduced to that of ElGamal encryption scheme. It is well known that ElGamal encryption scheme is indistinguishably secure against chosen plaintext attack under the DDH assumption. The details will be given in the final paper.

\subsection{Black Box Tracing Algorithm for LC-KD Scheme}

Let $B A D$ be the set of at most $k$ traitors who created a confiscated pirate decoder. Let $A$ be a subset of at most $k$ users. We first describe a procedure TEST which checks whether $A \cap B A D \neq \emptyset$.

Suppose that $\left(e_{T}, e_{1}, \cdots, e_{n}\right)$ is being used as the key. For a random encryption key $e_{T}^{\prime}=\left(a_{1}^{\prime}, \cdots, a_{n-m}^{\prime}\right)$, let the corresponding private decryption keys be $\left(e_{1}^{\prime}, \cdots, e_{n}^{\prime}\right)=\left(a_{1}^{\prime}, \cdots, a_{n-u}^{\prime}\right) H$. We say that $e_{T}^{\prime}$ matches with $A$ if $e_{i}^{\prime}=e_{i}$ for all $i \in A$.

$\operatorname{TEST}(A)$

Step 1. $T$ chooses $e_{T}^{\prime}$ which matches $A$ randomly. (We can do this because any $2 k$ columns of $H$ are linearly independent.) He chooses a random session key $s^{\prime}$ and computes an illegal header $h^{\prime} \stackrel{R}{\leftarrow} e_{T}^{\prime}\left(s^{\prime}\right)$. 
Step 2. $T$ gives $h^{\prime}$ to the pirate decoder. Let the output of the pirate decoder be $s_{A}$.

Output:

$$
\operatorname{TEST}(A)=\left\{\begin{array}{lc}
1 & \text { if } s_{A}=s^{\prime} \\
0 & \text { otherwise }
\end{array}\right.
$$

We next describe a procedure $\operatorname{TEST2}(A, m)$ which runs $T E S T(A) m$ times independently, where $m$ is a sufficiently large positive integer.

$\operatorname{TEST2}(A, m)$

Set counter $:=0$. For $i=1,2, \ldots, m$, do

Step 1. Run $T E S T(A)$ randomly.

Step 2. Let counter $:=$ counter $+\operatorname{TEST}(A)$. Reset the pirate decoder.

Output: $\operatorname{TEST2}(A, m)$, the final value of counter.

We say that a set of users $A$ is marked if $\operatorname{TEST2}(A, m)=m$. We now present our tracing algorithm.

Black box tracing algorithm

Find a marked set $A=\left\{i_{1}, i_{2}, \ldots, i_{k}\right\}$ by exhaustive search. Suppose that $i_{1}<$ $i_{2}<\cdots<i_{k}$. For $j=1,2, \ldots, k$, do:

Step 1. Let $B:=A \backslash\left\{i_{1}, i_{2}, \ldots, i_{j}\right\}$. Run $\operatorname{TEST2}(B, m)$.

Step 2. Let $m_{j}=T E S T 2(B, m)$.

Output: $i_{j}$ such that $m_{j-1}-m_{j}$ is the maximum. (If there are more than one such $j$, choose one of them arbitrarily.) User $i_{j}$ is a traitor.

Remark 7.1. By testing all the permutations on $\left\{i_{1}, i_{2}, \ldots, i_{k}\right\}$ (instead of $i_{1}<$ $i_{2}<\cdots<i_{k}$ ), we can detect all traitors who are active in $A$. All active traitors are found by applying this process to all marked sets $A$.

\subsection{Validity of Our Tracing Algorithm}

We can show the validity of our tracing algorithm by using the following three test conditions.

(1) If $A \supseteq B A D$, then $\operatorname{Pr}(T E S T(A)=1)$ is overwhelming.

(2) If $A \cap B A D=\emptyset$, then $\operatorname{Pr}(T E S T(A)=1)$ is negligible.

(3) If $A \cap B A D \neq \emptyset$ and $A \backslash B A D \neq \emptyset$, then for any $i \in A \backslash B A D$,

$$
|\operatorname{Pr}(T E S T(A)=1)-\operatorname{Pr}(T E S T(A \backslash\{i\})=1)|
$$

is negligible.

Theorem 7.2. If the above three conditions are satisfied, then our black box tracing algorithm succeeds with overwhelming probability. That is user $i_{j}$ is a traitor. 
Proof. If $A \supseteq B A D$, then TEST2 $(A, m)=m$ with overwhelming probability from (1). Therefore, there exists at least one marked $A$. On the other hand, from (2), if $A \cap B A D=\emptyset$, then $T E S T 2(A, m) \ll m$. This means that if $A$ is marked, then $A \cap B A D \neq \emptyset$.

Now suppose that $A$ is marked. Let $m_{0}=m$. It is easy to see that $m_{k}=0$. If $m_{j-1}-m_{j}$ is the maximum, then $m_{j-1}-m_{j} \geq m / k$. On the other hand, if $j \in A \backslash B A D$, then $m_{j-1}-m_{j} \ll m / k$ from (3). Therefore, if $m_{j-1}-m_{j}$ is the maximum, then $i_{j} \in B A D$.

We finally show that LC-KD scheme satisfies the above three test conditions under the DDH assumption. We assume that a pirate decoder decrypts valid headers with overwhelming probability.

Theorem 7.3 (Test Condition (1)). In LC-KD scheme, if $A \supseteq B A D$, then $\operatorname{Pr}(T E S T(A)=1)$ is overwhelming under the DDH assumption.

Theorem 7.4 (Test Condition (2)). In $L C-K D$ scheme, if $A \cap B A D=\emptyset$, then $\operatorname{Pr}(T E S T(A)=1)$ is negligible under the DDH assumption.

Theorem 7.5 (Test Condition (3)). In $L C-K D$ scheme, if $A \cap B A D \neq \emptyset$ and $A \backslash B A D \neq \emptyset$, then for any $i \in A \backslash B A D$,

$$
|\operatorname{Pr}(T E S T(A)=1)-\operatorname{Pr}(T E S T(A \backslash\{i\})=1)|
$$

is negligible under the DDH assumption.

The proofs will be given in Appendix.

\subsection{Secrecy and Traceability of LC-KD' Scheme}

The secrecy and the traceability of LC-KD' Scheme are proved similarly.

Theorem 7.6. LC-KD' scheme is indistinguishably secure against chosen plaintext attack under the DDH assumption.

Theorem 7.7 (Test Condition (1)). In $L C$-KD' scheme, if $A \supseteq B A D$, then $\operatorname{Pr}(T E S T(A)=1)$ is overwhelming under the DDH assumption.

Theorem 7.8 (Test Condition (2)). In LC-KD' scheme, if $A \cap B A D=\emptyset$, then $\operatorname{Pr}(T E S T(A)=1)$ is negligible under the DDH assumption.

Theorem 7.9 (Test Condition (3)). In $L C$-KD' scheme, if $A \cap B A D \neq \emptyset$ and $A \backslash B A D \neq \emptyset$, then for any $i \in A \backslash B A D$,

$$
|\operatorname{Pr}(T E S T(A)=1)-\operatorname{Pr}(T E S T(A \backslash\{i\})=1)|
$$

is negligible under the DDH assumption.

We show the proof of Theorem 7.8 in Appendix. The other theorems are proved similarly to those of LC-KD scheme. 


\subsection{Secrecy and Traceability of LC-BF Scheme}

The secrecy and the traceability of LC-BF Scheme are equivalent to those of LC-KD' scheme as shown in Sec6.1.

\section{References}

1. M. Bellare, A. Boldyreva and S. Micali, "Public-key encryption in a multi-user setting: Security proofs and improvements," Proceedings of EUROCRYPT 2000, LNCS 1807, Springer Verlag, pp.259-274, 2000.

2. D. Boneh and M. Franklin, "An efficient public key traitor tracing scheme (Extended Abstract)," Proceedings of CRYPTO '99, LNCS 1666, Springer Verlag, pp.338-353, 1999.

3. D. Boneh and M. Franklin, "An efficient public key traitor tracing scheme (fullversion of [2])," http://crypto.stanford.edu/ dabo/, 2001.

4. B. Chor, A. Fiat, and M. Naor, "Tracing traitors," Proceedings of CRYPTO '94, LNCS 839, Springer Verlag, pages 257-270, 1994.

5. B. Chor, A. Fiat, and M. Naor, B. Pinkas, "Tracing traitors," IEEE Transactions on Information Theory, Vol.46, No.3, pp.893-910, 2000.

6. K. Kurosawa and Y. Desmedt, "Optimum traitor tracing and asymmetric schemes with arbiter," Proceedings of EUROCRYPT '98, LNCS 1403, Springer Verlag, pp.145-157, 1999.

7. M. Naor and O. Reingold, "Number theoretic constructions of efficient pseudorandom functions," Proceedings of 38th IEEE Symposium on Foundations of Computer Science, pp.458-467, 1997.

8. M. Stadler, "Publicly verifiable secret sharing," Proceedings of EUROCRYPT '96, LNCS 1070, Springer Verlag, pp.190-199, 1996.

9. D. Stinson and R. Wei, "Combinatorial properties and constructions of traceability schemes and frameproof codes," SIAM Journal on Discrete Mathematics, Vol.11, No.1, pp.41-53, 1998.

10. D. Stinson and R. Wei, "Key preassigned traceability schemes for broadcast encryption," Proceedings of SAC'98, LNCS 1556, Springer Verlag, pp.144-156, 1998.

\section{A Proof of Theorem 7.3}

By extending the result of Stadler [8, in the proof of Proposition 1] and Naor and Reingold [7] lemma 3.2], Bellare et al. proved the following proposition [1].

Proposition A.1. [1] There is a probabilistic algorithm $\Sigma$ such that on input $g^{a}, g^{b}, g^{c}, \Sigma$ outputs $g^{b^{\prime}}, g^{c^{\prime}}$, where $b^{\prime}$ is random and

$$
c^{\prime}= \begin{cases}a b^{\prime} \bmod p & \text { if } c=a b \bmod p \\ r a n d o m & \text { if } c \neq a b \bmod p\end{cases}
$$

$\Sigma$ runs in $O\left(T^{e x p}\right)$ time, where $T^{e x p}$ is the time needed to perform an exponentiation. 
Now we show that

$$
p_{0}=\mid \operatorname{Pr}(P \text { decrypts valid headers correctly })-\operatorname{Pr}(T E S T(A)=1) \mid
$$

is negligible for any pirate decoder $P$.

Suppose that $p_{0} \geq \epsilon$ for some nonnegligible probability $\epsilon$. Then we show that there exists a probabilistic polynomial time Turing machine $M$ which can distinguish $\mathbf{D}=\left(g, g^{a}, y, y^{a}\right)$ and $\mathbf{R}=\left(g, g^{a}, y, v\right)$ with nonnegligible probability, where $g, y, v$ are chosen at random from $G_{q}$ and $a$ is chosen at random in $Z_{q}$.

From our assumption, there is an algorithm $B$ which creates a pirate decoder such that $p_{0} \geq \epsilon$ from a public key $p k=\left(g, y_{1}, \cdots, y_{2 k}\right)$ and the private keys of $B A D$.

Now on input $d=\left(g, g^{\prime} y, y^{\prime}\right), M$ works as follows.

1. Choose $e_{i}$ at random for each $i \in A$ and let $e_{i}^{\prime}=e_{i}$ for each $i \in A$.

2. Let $O U T=\left\{i_{1}, i_{2}, \ldots, i_{k}\right\}$ be a $k$-subset of users such that $O U T \cap A=\emptyset$.

3. For $j=1,2, \ldots, k, M$ runs $\Sigma$ of Proposition A.1 $k$ times independently on input $d=\left(g, g^{\prime}, y, y^{\prime}\right)$. Let the output of $\Sigma$ be $g^{e_{i_{j}}},\left(g^{\prime}\right)^{e_{i_{j}}^{\prime}}$.

4. Compute $g^{a_{1}}, g^{a_{2}}, \ldots, g^{a_{n-u}}$ from $\left\{g^{e_{i}} \mid i \in O U T \cup A\right\}$, where

$$
\left(e_{1}, \cdots, e_{n}\right)=\left(a_{1}, \cdots, a_{n-u}\right) H .
$$

Each $a_{i}$ is written as a linear combination of $\left\{e_{i} \mid i \in A \cup B A D\right\}$ because any $2 k$ columns of $H$ are linearly independent and $|A \cup B A D| \leq 2 k$. Therefore, we can do this.

5. Compute $\left(g^{\prime}\right)^{a_{1}^{\prime}},\left(g^{\prime}\right)^{a_{2}^{\prime}}, \ldots,\left(g^{\prime}\right)^{a_{n-u}^{\prime}}$ from $\left\{\left(g^{\prime}\right)^{e_{i}^{\prime}} \mid i \in O U T \cup A\right\}$, where

$$
\left(e_{1}^{\prime}, \cdots, e_{n}^{\prime}\right)=\left(a_{1}^{\prime}, \cdots, a_{n-u}^{\prime}\right) H .
$$

6. Select a random session key $s^{\prime}$ and compute $h^{\prime}$ as follows.

$$
h^{\prime}=\left(g^{\prime}, s^{\prime}\left(g^{\prime}\right)^{a_{1}^{\prime}},\left(g^{\prime}\right)^{a_{2}^{\prime}}, \ldots,\left(g^{\prime}\right)^{a_{n-u}^{\prime}}\right) .
$$

7. Create a pirate decoder $P$ by running $B$ on input a public key $\left(g, g^{a_{1}}, g^{a_{2}}, \ldots, g^{a_{n-u}}\right)$ and the private keys of $B A D,\left\{e_{i} \mid i \in B A D\right\}$.

8. Give $h^{\prime}$ to the pirate decoder $P$. Let the output of $P$ be $s_{A}$.

9. Finally $M$ outputs 1 if $s_{A}=s^{\prime}$ or 0 otherwise.

For $O U T=\left\{i_{1}, i_{2}, \ldots, i_{k}\right\}$, it holds that

$$
e_{i_{j}}^{\prime}= \begin{cases}e_{i_{j}} \bmod p & \text { if } d \leftarrow \mathbf{D} \\ \text { random } & \text { if } d \leftarrow \mathbf{R} .\end{cases}
$$

from Proposition A.1. Therefore, if $d$ is chosen from $\mathbf{D}, h^{\prime}$ is a legal header. On the other hand, if $d$ is chosen from $\mathbf{R}, h^{\prime}$ is an illegal header used in $\operatorname{TEST}(A)$. Hence, we have

$$
\begin{aligned}
\mid \operatorname{Pr}(M(d) & =1 \mid d \in \mathbf{D})-\operatorname{Pr}\left(M_{k}(d)=1 \mid d \in \mathbf{R}\right) \mid \\
& =p_{0} \\
& \geq \epsilon .
\end{aligned}
$$

from our assumption.

This means that $M$ can distinguish $\mathbf{D}$ and $\mathbf{R}$ with nonnegligible probability. 


\section{B Proof of Theorem 7.4}

Suppose that $\operatorname{Pr}(\operatorname{TEST}(A)=1) \geq \epsilon$ for some nonnegligible probability $\epsilon$. Then we show that there exists a probabilistic polynomial time Turing machine $M$ which can distinguish $\mathbf{D}=\left(g, g^{a}, y, y^{a}\right)$ and $\mathbf{R}=\left(g, g^{a}, y, v\right)$ with nonnegligible probability, where $g, y, v$ are chosen at random from $G_{q}$ and $a$ is chosen at random in $Z_{q}$.

From our assumption, there is an algorithm $B$ which creates a pirate decoder $P$ such that $\operatorname{Pr}(T E S T(A)=1) \geq \epsilon$ from a public key $p k=\left(g, y_{1}, \cdots, y_{2 k}\right)$ and the private keys of $B A D$.

Now on input $d=\left(g, g^{\prime}, y, y^{\prime}\right), M$ works as follows.

1. Choose $a_{2}^{\prime}, \ldots, a_{n-u}^{\prime}$ at random. Let $a_{1}^{\prime}$ be such that $g^{a_{1}^{\prime}}=y$.

2. Select a random session key $s^{\prime}$ and compute $h^{\prime}$ as follows.

$$
h^{\prime}=\left(g^{\prime}, s^{\prime} y^{\prime}, y^{a_{2}^{\prime}}, y^{a_{3}^{\prime}}, \ldots, y^{a_{n-u}^{\prime}}\right) .
$$

3. Compute $g^{e_{1}^{\prime}}, g^{e_{2}^{\prime}}, \ldots, g^{e_{n}^{\prime}}$ from $g^{a_{1}^{\prime}}, g^{a_{2}^{\prime}}, \ldots, g^{a_{n-u}^{\prime}}$, where

$$
\left(e_{1}^{\prime}, \cdots, e_{n}^{\prime}\right)=\left(a_{1}^{\prime}, \cdots, a_{n-u}^{\prime}\right) H .
$$

4. Choose $e_{i}$ at random for each $i \in B A D$. Let $g^{e_{i}}=g^{e_{i}^{\prime}}$ for each $i \in A$.

5. From $\left\{g^{e_{i}} \mid i \in A \cup B A D\right\}$, compute $g^{a_{1}}, g^{a_{2}}, \ldots, g^{a_{n-u}}$, where

$$
\left(e_{1}, \ldots, e_{n}\right)=\left(a_{1}, \cdots, a_{n-u}\right) \cdot H
$$

Each $a_{i}$ is written as a linear combination of $\left\{e_{i} \mid i \in A \cup B A D\right\}$ because any $2 k$ columns of $H$ are linearly independent and $|A \cup B A D| \leq 2 k$. Therefore, we can do this.

6. Create a pirate decoder $P$ by running $B$ on input a public key $\left(g, g^{a_{1}}, g^{a_{2}}, \ldots, g^{a_{n-u}}\right)$ and the private keys of $B A D,\left\{e_{i} \mid i \in B A D\right\}$.

Give $h^{\prime}$ to the pirate decoder $P$. Let the output of $P$ be $s_{A}$.

7. Finally $M$ outputs 1 if $s_{A}=s^{\prime}$ or 0 otherwise.

Then we obtain that

$$
\begin{aligned}
\mid \operatorname{Pr}(M(d) & =1 \mid d \leftarrow \mathbf{D})-\operatorname{Pr}(M(d)=1 \mid d \leftarrow \mathbf{R}) \mid \\
& =\left|\operatorname{Pr}\left(s_{A}=s^{\prime} \mid d \leftarrow \mathbf{D}\right)-\operatorname{Pr}\left(s_{A}=s^{\prime} \mid d \leftarrow \mathbf{R}\right)\right|
\end{aligned}
$$

First we see that $\operatorname{Pr}\left(s^{\prime}=s \mid d \leftarrow \mathbf{R}\right)$ is negligible because $y^{\prime}$ is random. Next it is easy to see that if $d$ is chosen from $\mathbf{D}$, then $h^{\prime}$ is a testing header used in $\operatorname{TEST}(A)$. Therefore,

$$
\operatorname{Pr}\left(s_{A}=s^{\prime} \mid d \leftarrow \mathbf{D}\right)=\operatorname{Pr}(T E S T(A)=1) \geq \epsilon
$$

from our assumption.

This means that $M$ can distinguishes $\mathbf{D}$ and $\mathbf{R}$ with nonnegligible probability. 


\section{Proof of Theorem 7.5}

Suppose that

$$
|\operatorname{Pr}(T E S T(A)=1)-\operatorname{Pr}(T E S T(A \backslash\{\tilde{i}\})=1)| \geq \epsilon
$$

for some nonnegligible probability $\epsilon$. Then we show that there exists a probabilistic polynomial time Turing machine $M$ which can distinguish $\mathbf{D}=\left(g, g^{r}, y, y^{r}\right)$ and $\mathbf{R}=\left(g, g^{r}, y, v\right)$ with nonnegligible probability, where $g, y, v$ are chosen at random from $G_{q}$ and $r$ is chosen at random from $Z_{q}$.

From our assumption, there is an algorithm $B$ which creates a pirate decoder $P$ such that

$$
|\operatorname{Pr}(T E S T(A)=1)-\operatorname{Pr}(T E S T(A \backslash\{i\})=1)| \geq \epsilon
$$

from a public key $p k=\left(g, y_{1}, \ldots, y_{n-u}\right)$ and the private keys of $B A D$.

Now on input $d=\left(g, g^{\prime}, y, y^{\prime}\right), M$ works as follows.

1. Choose $e_{i}$ for each $i \in B A D \cup(A \backslash\{\tilde{i}\})$. Let $e_{\tilde{i}}$ be such that $g^{e^{\tilde{i}}}=y$.

2. Compute $g^{a_{1}}, g^{a_{2}}, \ldots, g^{a_{n-u}}$ from $\left\{g^{e_{i}} \mid i \in B A D \cup A\right\}$, where

$$
\left(e_{1}, \cdots, e_{n}\right)=\left(a_{1}, \cdots, a_{n-u}\right) H
$$

3. Create a pirate decoder $P$ by running $B$ on input a public key $\left(g, g^{a_{1}}, g^{a_{2}}, \ldots, g^{a_{n-u}}\right)$ and the private keys of $B A D,\left\{e_{i} \mid i \in B A D\right\}$.

4. Next let $e_{i}^{\prime}=e_{i}$ for each $i \in A \backslash\{\tilde{i}\}$ and $e_{\tilde{i}}^{\prime}$ be such that $y^{e_{\tilde{i}}^{\prime}}=y^{\prime}$.

5. Compute $y^{a_{1}^{\prime}}, y^{a_{2}^{\prime}}, \ldots, y^{a_{n-u}^{\prime}}$ from $\left\{y^{e_{i}^{\prime}} \mid i \in B A D \cup A\right\}$, where

$$
\left(e_{1}^{\prime}, \cdots, e_{n}^{\prime}\right)=\left(a_{1}^{\prime}, \cdots, a_{n-u}^{\prime}\right) H .
$$

6. Select a random session key $s^{\prime}$ and compute $h^{\prime}$ as follows.

$$
h^{\prime}=\left(g^{\prime}, s^{\prime} y^{a_{1}^{\prime}}, y^{a_{2}^{\prime}}, \ldots, y^{a_{n-u}^{\prime}}\right)
$$

Give $h^{\prime}$ to the pirate decoder $P$. Let the output of $P$ be $s_{A}$.

7. Finally $M$ outputs 1 if $s_{A}=s^{\prime}$ or 0 otherwise.

It is easy to see that if $d$ is chosen from $\mathbf{D}$, then $h^{\prime}$ is an illegal header used in $\operatorname{TEST}(A)$. On the other hand, if $d$ is chosen from $\mathbf{R}$, then $h^{\prime}$ is an illegal header used in $\operatorname{TEST}(A \backslash\{\tilde{i}\})$.

Therefore,

$$
\begin{aligned}
\mid \operatorname{Pr}(M(d) & =1 \mid d \leftarrow \mathbf{D})-\operatorname{Pr}(M(d)=1 \mid d \leftarrow \mathbf{R}) \mid \\
& =|\operatorname{Pr}(T E S T(A)=1)-\operatorname{Pr}(T E S T(A \backslash\{\tilde{i}\})=1)| \\
& \geq \epsilon
\end{aligned}
$$

from our assumption.

This means that $M$ can distinguish $\mathbf{D}$ and $\mathbf{R}$ with nonnegligible probability. 


\section{Proof of Theorem 7.8}

Suppose that $\operatorname{Pr}(T E S T(A)=1) \geq \epsilon$ for some nonnegligible probability $\epsilon$. Then we show that there exists a probabilistic polynomial time Turing machine $M$ which can distinguish $\mathbf{D}=\left(g, g^{a}, y, y^{a}\right)$ and $\mathbf{R}=\left(g, g^{a}, y, v\right)$ with nonnegligible probability, where $g, y, v$ are chosen at random from $G_{q}$ and $a$ is chosen at random in $Z_{q}$.

From our assumption, there is an algorithm $B$ which creates a pirate decoder such that $\operatorname{Pr}(T E S T(A)=1) \geq \epsilon$ from a public key $p k=\left(g, y_{1}, \cdots, y_{2 k}\right)$ and the private keys of $B A D$.

Now on input $d=\left(g, g^{\prime}, y, y^{\prime}\right), M$ works as follows.

1. Choose $e_{i}$ at random for each $i \in B A D$.

2. For each $i \in A$, choose $t_{i}$ at random and compute $y^{t_{i}}$. Define $e_{i}$ as $g^{e_{i}}=y^{t_{i}}$.

3. From $\left\{g^{e_{i}} \mid i \in A \cup B A D\right\}$, compute $g^{a_{1}}, g^{a_{2}}, \ldots, g^{a_{n-u}}$, where

$$
\left(e_{1}, \ldots, e_{n}\right)=\left(a_{1}, \cdots, a_{n-u}\right) \cdot H .
$$

4. Create a pirate decoder $P$ by running $B$ on input a public key $\left(g, g^{a_{1}}, g^{a_{2}}, \ldots, g^{a_{n-u}}\right)$ and the private keys of $B A D,\left\{e_{i} \mid i \in B A D\right\}$.

5. For each $i \in A$, compute $\beta_{i}=\left(y^{\prime}\right)^{t_{i}}$. For each $i \in B A D$, choose a random element $\beta_{i}$.

6. Suppose that $y^{\prime}=y^{r}$. Define $e_{i}^{\prime}$ as $\beta_{i}=g^{r e_{i}^{\prime}}$ for each $i \in(A \cup B A D)$.

7. From $\left\{\beta_{i} \mid i \in A \cup B A D\right\}$, compute $g^{r a_{1}^{\prime}}, g^{r a_{2}^{\prime}}, \ldots, g^{r a_{n-u}^{\prime}}$, where $\beta_{i}=g^{r e_{i}^{\prime}}$ and

$$
\left(e_{1}^{\prime}, \ldots, e_{n}^{\prime}\right)=\left(a_{1}^{\prime}, \cdots, a_{n-u}^{\prime}\right) \cdot H \text {. }
$$

8. Select a random session key $s^{\prime}$ and compute $h^{\prime}$ as follows.

$$
h^{\prime}=\left(s^{\prime} g^{\prime}, g^{r a_{1}^{\prime}}, g^{r a_{2}^{\prime}}, \ldots, g^{r a_{n-u}^{\prime}}\right) \text {. }
$$

Give $h^{\prime}$ to the pirate decoder $P$. Let the output of $P$ be $s_{A}$.

9. Finally $M$ outputs 1 if $s_{A}=s^{\prime}$ or 0 otherwise.

Then we obtain that

$$
\begin{aligned}
\mid \operatorname{Pr}(M(d) & =1 \mid d \leftarrow \mathbf{D})-\operatorname{Pr}(M(d)=1 \mid d \leftarrow \mathbf{R}) \mid \\
& =\left|\operatorname{Pr}\left(s_{A}=s^{\prime} \mid d \leftarrow \mathbf{D}\right)-\operatorname{Pr}\left(s_{A}=s^{\prime} \mid d \leftarrow \mathbf{R}\right)\right|
\end{aligned}
$$

First we see that $\operatorname{Pr}\left(s^{\prime}=s \mid d \leftarrow \mathbf{R}\right)$ is negligible because $g^{\prime}$ is random.

Next we will show that if $d$ is chosen from $\mathbf{D}$, then $h^{\prime}$ is an illegal header used in $\operatorname{TEST}(A)$. In this case, $y^{\prime}=y^{r}$ and $g^{\prime}=g^{r}$ for some $r$. We need to show that $e_{i}^{\prime}=e_{i}$ for each $i \in A$. Assume that $y=g^{x}$. Then

1. $e_{i}=x t_{i}$ since $y^{t_{i}}=g^{e_{i}}$.

2. On the other hand,

$$
g^{r e_{i}^{\prime}}=\beta_{i}=\left(y^{\prime}\right)^{t_{i}}=y^{r t_{i}}=g^{x r t_{i}} .
$$

Therefore, $e_{i}^{\prime}=x t_{i}$.

Hence, $e_{i}^{\prime}=e_{i}$. Therefore, $h^{\prime}$ is an illegal header used in $\operatorname{TEST}(A)$. Consequently,

$$
\operatorname{Pr}\left(s_{A}=s^{\prime} \mid d \leftarrow \mathbf{D}\right)=\operatorname{Pr}(T E S T(A)=1) \geq \epsilon
$$

from our assumption.

This means that $M$ can distinguishes $\mathbf{D}$ and $\mathbf{R}$ with nonnegligible probability. 